\section{"É muito dificultoso!": etnografia dos cuidados a pacientes com hipertensão e/ou diabetes na atenção básica, em Manaus, Amazonas, Brasil}

\author{
"It sure ain't easy!": an ethnographic study of \\ primary health care for patients with hypertension \\ and/or diabetes in Manaus, Amazonas State, Brazil
}

\author{
${ }^{1}$ Centro de Pesquisa Leônidas \\ e Maria Deane, Fundação \\ Oswaldo Cruz \\ Manaus, Brasil. \\ 2 Universidade Federal do \\ Amazonas, Manaus, Brasil. \\ Correspondência \\ M. L. P. Souza \\ Centro de Pesquisa Leônida \\ e Maria Deane, Fundação \\ Oswaldo Cruz. \\ Rua Teresina 476, Manaus, \\ AM 69057-070, Brasil. \\ maximiliano@amazonia. \\ fiocruz.br
}

\begin{abstract}
This paper analyzes the results of an evaluative study in the city of Manaus, Amazonas State, Brazil, on primary health care for patients with hypertension and/or diabetes. The ethnographic approach used access to services and comprehensiveness of health care as core analytical categories, comparing the health practices developed by Family Health Program (FHP) units with traditional non-FHP primary care units. Access to family health care units in low-income communities is limited by the precarious surrounding urban infrastructure. The main barrier to access to primary care units is distance. The lack of a referral system between the various levels of complexity jeopardizes patients' access to tests and specialists. The care supplied by the two units is limited to patient conditions that can be treated pharmacologically, thus compromising the comprehensiveness of care. The health professionals display a limited capacity to hear problems outside the immediate focus of the program activity. The paper highlights the potential for using ethnography in evaluative research on health systems and services.
\end{abstract}

Primary Heath Care; Cultural Anthropology; Hypertension; Diabetes Mellitus
Maximiliano Loiola Ponte de Souza 1

Luíza Garnelo 1,2

\section{Introdução}

O envelhecimento populacional apresenta-se como um fenômeno global 1, associando-se ao aumento da morbi-mortalidade por doenças cardiovasculares 2 . A hipertensão arterial sistêmica e o diabetes mellitus são agravos independentes e freqüentemente sinérgicos cuja combinação redunda em grave comprometimento à saúde. Ambos necessitam de acompanhamento a longo prazo; exigem mudança de hábitos ${ }^{3,4} \mathrm{e}$, por vezes, o uso de medicação por toda a vida.

O Ministério da Saúde 5, ao propor o Plano de Reorganização da Atenção à Hipertensão Arterial Sistêmica e ao Diabetes Mellitus, reconhece a importância da Atenção Básica, na abordagem desses agravos, feita por meio do modelo de atenção programática denominada HIPERDIA (Sistema de Cadastramento e Acompanhamento de Hipertensos e Diabéticos) que possibilita o desenvolvimento de ações contínuas e de alta capilaridade. Sugere que o cuidado ofertado deva ir além do binômio queixa-conduta, que permita identificar assintomáticos, monitorar o tratamento, estabelecer vínculos entre equipe de saúde-pacientes-cuidadores e realizar educação em saúde, incorporando a realidade social do paciente a esse processo.

$\mathrm{Na}$ implementação da Atenção Básica no Brasil, o Programa Saúde da Família (PSF) se destacou como uma via de extensão de cobertura e de vigilância em saúde dirigida a grupos em 
situação de vulnerabilidade. Nos últimos anos, o programa foi redimensionado na forma de um conjunto de ações estratégicas capazes de reordenar o modelo assistencial e viabilizar a integração entre as diversas formas de cuidados e níveis de atenção 6,7, no Sistema Único de Saúde (SUS). Para viabilizar tal objetivo, diversas atividades, dentre as quais, uma pesquisa avaliativa das ações do programa nos municípios com mais de 100 mil habitantes, foram desenvolvidas em todo o território nacional, sob a coordenação do Projeto de Expansão e Consolidação do Saúde da Família (PROESF), voltado para o apoio à reestruturação da Estratégia Saúde da Família. Na Amazônia ocidental, a investigação foi realizada em cinco municípios: Manaus, Boa Vista, Rio Branco, Porto Velho e Ji-Paraná.

Os dados que dão origem a este artigo são parte dessa pesquisa de linha de base, produzidos a partir de uma etnografia das práticas sanitárias da atenção pré-natal e do programa de controle da hipertensão e do diabetes mellitus (HIPERDIA) na rede básica do Município de Manaus. O componente antropológico foi desenvolvido ao longo de oito meses, entre os anos de 2005-2006. As categorias analíticas centrais do estudo foram o acesso aos serviços e a integralidade do cuidado na rede básica de saúde. Na investigação, as supracitadas formas de atenção programáticas foram consideradas como condições traçadoras, aqui entendidas como um meio de "sinalizar, monitorar ou evidenciar aspectos substantivos da qualidade de serviços de saúde" 8 (p. 536). O presente texto efetua a análise das informações coletadas junto ao programa HIPERDIA. Os dados sobre atenção pré-natal serão discutidos em outra publicação.

\section{Dimensões organizativas da Atenção Básica à Saúde no Município de Manaus}

Em 2005, Manaus tinha uma população estimada em 1.644.688 habitantes. O grupo etário com 60 anos ou mais correspondia a $4,67 \%$ do total da população. Tal número representava um incremento de $17,33 \%$ nessa faixa etária, em comparação com valores de vinte anos atrás. No mesmo período, a população menor de 15 anos sofreu uma redução de $17 \%$. Acompanhando a tendência de envelhecimento populacional, observouse que, em 2005, 6,11\% das internações hospitalares no SUS foram geradas por doenças do aparelho circulatório, um valor 1,8 vez maior do que o ocorrido em 1995. Em 2004, a mortalidade proporcional por doenças do aparelho circulatório foi de $17,8 \%$, em contraponto aos $14,7 \%$ de 1984 , o que representou um incremento de $21 \%$.
Manaus encontra-se habilitada na gestão plena da atenção básica desde 2003. Na rede municipal de saúde, coexistiam, na época da coleta de dados, unidades básicas de saúde com e sem-PSF; essas últimas atendiam à demanda espontânea, não operando em bases territoriais. Parte das unidades não-PSF possuía o Programa de Agentes Comunitários de Saúde (PACS). Persistiam áreas descobertas por ambos os modelos assistenciais.

De acordo com o Sistema de Informação da Atenção Básica (SIAB), em 2005, havia, em Manaus, 655.999 pessoas cadastradas no PSF. Tal número expressava uma cobertura de apenas 39,9\% da população-alvo do programa estimada para $70 \%$ da população nos municípios com mais de 100 mil habitantes. De acordo com a norma técnica, cada equipe PSF deve acompanhar, em média, 3.450 pessoas 9 ; nesse caso, o PSF, em Manaus, necessitaria de 190 equipes, mas tinha apenas 173 atuando no período da pesquisa. Considerando-se completas as equipes que possuíssem um médico, um enfermeiro, dois auxiliares de enfermagem e um número de agentes comunitários de saúde equivalente ao número de micro-áreas de abrangência da unidade, apenas $34,1 \%$ do total de equipes atuantes preenchiam esse critério.

\section{Metodologia}

O marco teórico e as estratégias de investigação foram únicos para todo o componente etnográfico, variando apenas a análise realizada para cada condição traçadora. A investigação, de tipo avaliativo, pautou-se pela proposta de Contandriopoulus et al. 10, para os quais este tipo de estudo exige a produção de julgamento de uma intervenção a partir de métodos e procedimentos científicos. A etnografia foi caracterizada como uma "pesquisa de campo com observação prolongada do objeto de estudo, seguida pela produção de dados em condições discursivas e dialógicas expressos através de formas textualizadas" 11 (p. 67); ela foi a via de abordagem qualitativa do cenário da pesquisa, "caracterizando-se pela íntima e dinâmica interação entre o observador e o objeto" 12 (p. 142) e pelo "status central dado à interpretação do significado das ações sociais" 13 (p. 106). Esta investigação deve ser considerada como um estudo de caso, aqui entendido como um sistema de relações instituídas em torno de uma prática social 14 que permitiu inferir propriedades gerais do modus operandi da Atenção Básica à Saúde no Município de Manaus.

A escolha do HIPERDIA como condição traçadora levou em consideração ser esse, de acor- 
do com a avaliação dos gerentes municipais de saúde, um programa efetivamente implantado na rede básica de Manaus; a influência da hipertensão arterial sistêmica e do diabetes mellitus no perfil de morbi-mortalidade no país ${ }^{2}$ e as evidências que o adequado controle dessas condições impacta positivamente nesses indicadores de saúde 5 .

O acesso como uma das categorias analíticas da pesquisa foi enfocado como um dos elementos-chave da organização dos sistemas de saúde, aqui abrangendo os aspectos referentes à entrada no serviço de saúde e à continuidade dos cuidados prestados 15. A integralidade foi abordada como uma forma totalizante de organização das práticas sanitárias que busca atender as necessidades de saúde dos usuários do SUS 16. Esse componente priorizou a investigação do acolhimento, responsabilização, vínculo e escuta nos serviços oferecidos na atenção básica 17 .

O trabalho de campo foi desenvolvido em uma unidade de saúde do PSF e em outra unidade básica de saúde não-PSF. A perspectiva comparativa foi priorizada devido à existência de formas competitivas de "modelos [que] atendem a populações-alvo similares e representam a porta de entrada ao sistema para os atendimentos eletivos" 8 (p. 536) na oferta de Atenção Básica no Município de Manaus. A seleção das unidades foi definida em conjunto com os gestores do município, por representarem unidades típicas do sistema que desenvolviam o HIPERDIA e pertencerem a uma mesma área geográfica, propiciando o entendimento de interações complementares ou competitivas entre elas.

A etnografia realizada propiciou uma "descrição densa” da realidade social e a integração de informações de diversos atores sociais que atuam numa mesma micro-realidade, palco de interações e conflitos 13,18. Dentre as técnicas de pesquisa utilizadas, realizou-se a observação participante das ações do HIPERDIA nos dois tipos de unidades de saúde, etnografando o atendimento médico, de enfermagem, as rotinas de pré e pós-consultas e o deslocamento de pacientes selecionados, desde seus domicílios até as unidades de saúde, assim como o trajeto inverso. Complementarmente realizaram-se grupos focais e entrevistas com profissionais e usuários da atenção programática investigada. A apresentação e discussão dos resultados foram feitas de modo comparativo, alternando-se os dados obtidos nos dois tipos de unidades pesquisadas.

\section{Resultados e discussão}

A unidade de saúde da família estudada localizase em uma área de invasão, caracterizada pela precariedade sócio-sanitária. A implantação desse serviço representou uma extensão de cobertura e viabilizou a prestação de ações de saúde a uma população anteriormente excluída. Porém, a carência de outros meios de infra-estrutura urbana compromete o acesso da clientela aos serviços ofertados na unidade PSF.

“Descendo a ladeira enlameada, a paciente escorregava bastante. Resolvemos que uma pessoa iria à frente e outra a apoiaria, segurando seu braço. Ela escorregou três vezes. (...) A sensação que tive era de estar participando de uma operação de resgate do Corpo de Bombeiros".

O trecho acima, oriundo do diário de campo dos pesquisadores, descreve a experiência de acompanhar uma paciente hipertensa e seqüelada de acidente vascular cerebral no percurso entre sua casa e a unidade de saúde da família. Sob a chuva, vencer as íngremes ladeiras de uma rua enlameada é o primeiro obstáculo - sobretudo para os idosos - a ser superado para acessar o atendimento. Soma-se a essa dificuldade a baixa cobertura do PSF no município, fazendo com que grandes áreas urbanas tenham as unidades do PSF como o único serviço de saúde disponível. Em conseqüência, além de penosas, as caminhadas podem ser longas.

Já a unidade básica de saúde conta com arredores urbanizados, mas sua localização em área portuária, com tráfico de drogas e prostituição nos arredores também gera dificuldade de acesso à unidade. O serviço não opera em bases territoriais. Isso não seria problema para os usuários circunvizinhos, caso não houvesse um grande número de clientes oriundos de outros locais da cidade, e até do interior do estado, atraídos pela oferta de exames complementares de diagnóstico e de especialistas como cardiologista, ginecologista e pediatra. A unidade dista mais de um quilômetro do principal aglomerado de domicílios do bairro. A inexistência de linha de ônibus que interligue as residências à unidade obriga os usuários a enfrentarem essa distância a pé.

$\mathrm{O}$ atendimento feito essencialmente por meio da demanda espontânea e o afluxo de grande número de usuários de diversas origens geravam pressão de demanda, dificultando o acesso aos cuidados. A falta de pré-marcação exigia que os usuários saíssem de casa antes do amanhecer, expondo-se à violência urbana para chegar bem cedo à unidade de saúde e obter consulta. Ressalte-se que a clientela oriunda da unidade de saúde da família mais próxima, onde também coletamos dados para a pesquisa, não obtinha 
atendimento preferencial em suas demandas à unidade básica de saúde. Tais fatores comprometiam a regularidade do contato dos pacientes com os serviços, inviabilizando a prevenção de complicações e o adequado controle da hipertensão arterial e do diabetes mellitus 19,20.

Na unidade de saúde da família, durante o período da pesquisa, observou-se que a baixa cobertura e a forte pressão de demanda tendiam a restringir a ação da equipe às atividades no interior da unidade, com prioridade para as consultas médico-curativas. A coordenação do PSF tinha uma atitude ambígua a esse respeito, havendo uma divisão de opiniões sobre a pertinência (ou não) da visita domiciliar como instrumento prioritário de ação. Tal condição refletia-se no trabalho local das equipes, reforçando a tendência espontânea dos profissionais de nível superior de se limitarem às ações intramuros, particularmente pelo temor da violência urbana.

A etnografia do cuidado flagrou a recomendação a um idoso com hipertensão severa com quadro de angina instável e histórico de acidente vascular cerebral para que comparecesse à unidade, duas vezes por semana, para aferição de sua pressão arterial. De acordo com o médico da unidade de saúde da família, tal procedimento permitiria um melhor controle da pressão arterial do paciente e o estimularia a desenvolver atividade física. O usuário "confidenciou" aos pesquisadores que não seguiria essa recomendação porque não teria como "vencer as ladeiras". No controle desses agravos, o exercício físico é uma medida não-farmacológica recomendada tanto para pacientes hipertensos quanto para diabéti$\cos ^{3}$, entretanto, ele deverá ter um caráter regular e com intensidade adequada à condição clínica do doente, sob pena de agravar seu quadro 21 .

Distorções como essas podem estar ligadas à falta de capacitação dos profissionais que atuam orientados apenas pelo seu senso comum e pela precariedade operacional do programa que não disponibilizava meios adequados para a avaliação correta dos casos clínicos e nem para efetivação das visitas domiciliares. Dá-se, então, uma reinterpretação da legislação que ampara o idoso, das normas técnicas do programa HIPERDIA e do próprio modelo de atenção preconizado pelo programa de saúde da família, levando os profissionais a priorizarem o atendimento biomédico individual em detrimento de ações de promoção à saúde.

As rotinas de trabalho do enfermeiro da unidade de saúde da família não previam agendamento de visitas domiciliares; eram realizadas "de acordo com a necessidade". Na época da pesquisa, essa atividade estava comprometida pela carência de profissionais na unidade, uma vez que o enfermeiro cobria as tarefas da auxiliar de enfermagem que, por ser estudante universitária e estar em período de estágio, comparecia ao trabalho uma única vez na semana; ele também realizava consultas quando o médico se ausentava da unidade. No entendimento do médico, as saídas do enfermeiro da unidade comprometiam o suporte que o mesmo deveria oferecer às consultas médicas.

"Antes da chegada do médico à unidade, o enfermeiro sai em seu carro para visitar uma senhora diabética e cega. Um pouco mais tarde, o médico inicia o atendimento do HIPERDIA, ignorando a saída do enfermeiro. Reclama que não foram calculados os índices de massa corporal dos pacientes; sob sua mesa há uma calculadora e acima dela a fórmula e os valores de referência para o cálculo do índice de massa corporal. Era patente seu desconforto com a situação, visto que 'não tinha ninguém para auxiliá-lo'. O auxílio demandado consistia em trazer para o consultório amostras de remédios que orientassem os pacientes sobre a posologia ou em informar os usuários sobre possiveis locais de realização de exames e de acesso à especialistas. Para o médico, o trabalho preferencial do enfermeiro devia ser o auxílio à consulta médica" (diário de campo - observação do atendimento).

Evidências na literatura apontam que a atividade de enfermeiros de saúde pública pautar-se-ia por uma subordinação epistêmica ao modelo médico 22. Na situação investigada, essa subordinação ia além. O enfermeiro incorporava as atribuições de outros profissionais ausentes, propondo-se a suprir as lacunas que ocorriam nas rotinas do serviço. Ele parecia ter dificuldade em delimitar um espaço próprio de atuação e em demonstrar, para o médico, a especificidade de suas tarefas que, certamente, ultrapassavam a mera função de "auxiliar de consultório".

A subalternização profissional alia-se à baixa capacidade resolutiva da atividade domiciliar, como se pode observar no relato abaixo:

"Retornando da visita, o enfermeiro relatou que a glicemia da paciente visitada estava 'acima de 500'. Sua conduta frente ao caso foi orientar que a paciente acionasse o serviço de ambulâncias de resgate domiciliar para ser levada a um pronto-socorro. Ele próprio não fez contato com a unidade de emergência, não definiu o serviço a ser procurado e nem efetuou o encaminhamento por escrito; tampouco aguardou, no domicílio, a chegada da ambulância. De acordo com o enfermeiro, sua conduta é um procedimento rotineiro, pois a equipe do PSF não dispõe de contatos que viabilizem a internação de emergência de doentes atendidos pelo programa, cabendo tal ônus aos clientes. Enunciou sua própria descrença no 
resgate dessa paciente e considerou que sua permanência no domicílio pouco contribuiria para uma maior resolutividade do problema. Aparentemente não lhe ocorreu acionar o médico da unidade PSF para insulinizar a paciente" (diário de campo).

Ao focalizar-se nas situações "problemáticas", a visita domiciliar do enfermeiro perde seu caráter preventivo. Deparando-se com situações que extrapolam a complexidade do nível básico de atenção, seus esforços mostram-se pouco resolutivos. Fica igualmente patente a inexistência de mecanismos de articulação da rede do PSF com os níveis mais especializados de atendimento.

Na unidade básica de saúde, a visita domiciliar aos pacientes do HIPERDIA por profissional de nível superior, embora possível, dada a existência do programa de agentes comunitários de saúde na unidade, padece de diversas limitações. Como parcela importante dos pacientes hipertensos e diabéticos ali atendidos reside em locais distantes, sua visitação é inviável. Para os que residem nas proximidades, a atividade é prejudicada pela não-delimitação de área de abrangência da unidade, que se superpõe à área de atuação de outra unidade básica de saúde existente no bairro. Ambas disputam a exclusividade do registro da clientela em suas respectivas estatísticas e sistema de cadastro sem que isso redunde em melhoria da cobertura ou da qualidade da atenção.

Os pacientes do HIPERDIA que consigam adentrar ao serviço serão atendidos na unidade de saúde da família, em um único dia da semana, pelo médico da família. Na unidade básica de saúde, há consulta diária de cardiologista e de clínico geral. Aqui, não há critério definidor de acompanhamento do paciente pelo clínico ou pelo especialista, isso depende exclusivamente da compatibilidade entre o horário do profissional e a vinda do paciente à unidade. Caso chegue no horário matutino, terá acesso ao especialista, do contrário, será atendido pelo clínico. Não há um sistema de triagem para maximizar a atividade do cardiologista; sua mão-de-obra é subutilizada e, muitas vezes, esse recebe pacientes com outras patologias apenas para completar sua cota de dezesseis atendimentos por turno de trabalho. Ambas as unidades oferecem atendimento de enfermagem para essa clientela.

O cuidado ofertado é centrado no indivíduo doente e focaliza aspectos biomédicos, sobretudo farmacológicos para o controle da hipertensão arterial e do diabetes mellitus. $\mathrm{O}$ atendimento médico relatado abaixo foi observado na unidade básica de saúde e será utilizado como fio condutor para discutir características dos cuidados oferecidos aos pacientes do HIPERDIA, na Atenção Básica de Manaus.
“A paciente 'S' começa a consulta relatando que entrou no climatério há três meses, que agora sente 'um calorão' e sua pressão disparou: 'antes era 12, depois passou para 14 e agora está em 18'. O médico registra essas informações no prontuário e, em seguida, inicia um 'diálogo': 'A senhora fuma? - Não. Faz atividade física? - Agora comecei a caminhar". Ele lê o prontuário da paciente para o pesquisador; recita: 'paciente virgem de tratamento, com história familiar de hipertensão, alegando estar no climatério, após o que teria ocorrido a elevação de pressão arterial'. Na seqüência, comenta que não costuma procurar dados familiares e sociais no prontuário, pois isso pode interessar para pesquisa, mas, na rotina, não têm importância, porque a hipertensão atinge pobres e ricos. Prossegue falando que, 'nesses casos virgens de tratamento', costuma recomendar dieta e atividade física, mas, como a paciente é hipertensa classe III, prescreverá medicação. Solicita um Eletrocardiograma e bioquímica de rotina. Define o esquema terapêutico: 'você vai tomar captopril de 12 em 12 horas e passiflorine de 8 em 8 horas"” (diário de campo).

É perceptível que o profissional não valorizou os sintomas de climatério, mesmo sendo essa uma condição associada à gênese e progressão de cardiopatias 23 . Seu foco se manteve na prescrição de anti-hipertensivo. Aparentemente, ele também interpretou as queixas da paciente como um quadro de ansiedade, condição apontada pela literatura como potencialmente relacionada à hipertensão arterial sistêmica 24 . Porém, a prescrição de um fitoterápico, sem eficácia terapêutica comprovada como ansiolítico 25 , é pouco promissora. O especialista demonstrou desconhecer as normas técnicas do programa e alegou preferência em seguir sua experiência; após algum tempo de conversa, admitiu nunca ter lido as normas técnicas do programa HIPERDIA.

A focalização em alguns aspectos biológicos da hipertensão arterial e do diabetes mellitus não é uma exclusividade da unidade básica de saúde. Na unidade de saúde da família pesquisada, uma paciente do HIPERDIA procurou o serviço com queixa de dificuldade visual. Foi orientada a retornar em outro momento, pois aquele era "o dia do HIPERDIA" e só, no dia seguinte, haveria atendimento de clínica geral. $\mathrm{O}$ evento apreende a dificuldade de articulação de queixas, mesmo no interior de uma única ação programática, tendo-se ignorado a associação recorrente entre diabetes e comprometimento visual 26 , que leva à recomendação de que os diabéticos se submetam à investigação periódica da saúde ocular. De forma similar, não observamos, durante as consultas, preocupação com outras ações preventivas, tais como a indicação de exames col- 
pocitológicos, mamografia ou outros meios de monitoramento da saúde do idoso.

Em ambas as unidades, o exame físico mostrou-se um procedimento excepcional. Após a coleta sumária das queixas, os médicos efetuavam a prescrição medicamentosa. Na unidade básica de saúde, o cardiologista realizava um exame físico simplificado e apressado. Tal atitude parecia inusitada para os pacientes que mostravam surpresa quando o médico se encaminhava em sua direção para examiná-los; alguns perguntavam como proceder durante o exame, levando-nos a concluir que tais atitudes do médico decorriam da presença dos pesquisadores no consultório e não de uma rotina de trabalho. Nossos achados corroboram os de Paiva et al. 2, no Município de Francisco Monorato, em São Paulo, que demonstraram as deficiências na realização desses procedimentos no PSF daquele município.

A desvalorização do conhecimento das condições de vida dos usuários e suas repercussões no tratamento representaram entraves para a adequada abordagem dos agravos controlados pelo HIPERDIA 1,20,27. Essas variáveis implicavam negativamente no uso regular de medicamentos pelos pacientes que moram em locais distantes da unidade cujos limites de acesso dificultavam o retorno às consultas. Os pesquisadores também observaram dificuldades no seguimento das dietas prescritas, seja pela falta de poder aquisitivo para adquirir os alimentos recomendados ou pelo significado simbólico e afetivo da partilha de refeições em família prejudicada pelo rigor de uma dieta "sem graça". Tais contextos eram ignorados pelos profissionais que atribuíam os problemas nessa esfera, exclusivamente, à falta de compromisso dos doentes com o tratamento.

Identificou-se, ainda, um importante erro de foco nas prescrições dietéticas, pois a recomendação de restrição de sal, de açúcar e de gordura era dirigida aos idosos, mas a pesquisa evidenciou que as jovens mulheres das famílias, pouco ou nada sensibilizadas para a importância da dieta, costumavam ser as encarregadas do preparo das refeições. Nessas circunstâncias, a retórica da equipe de saúde era desperdiçada com o idoso destituído de poder de decisão sobre as formas de preparo da comida. Conselhos e informações técnicas não alcançavam aquelas que poderiam contribuir para a redução do risco de complicação da hipertensão arterial e diabetes mellitus.

Os agentes comunitários de saúde da unidade de saúde da família detinham não apenas um amplo conhecimento do cenário social em que vivia a clientela, mas também reconheciam sua importância. Em um dos depoimentos, um agen- te comunitário se posicionou: “Às vezes, o médico diz que não pode comer certas coisas ou que tem que comer certo tipo de comidas para fazer dieta, mas os pacientes não têm como fazer isso. Como eu conheço a vida deles sei o que eles conseguem e o que não conseguem comer, e posso explicar isso para o médico". De acordo com o observado, os médicos pouco interesse demonstraram por tal conhecimento. O maior contato dos enfermeiros com os agentes comunitários de saúde favorecia a partilha dessas informações; porém, em ambos os tipos de unidade, os enfermeiros não conseguiam incorporá-las às suas rotinas de atendimento. $\mathrm{O}$ depoimento de enfermeira da unidade básica de saúde reforçou essa idéia: "Vemos a miséria horrível em que eles vivem; os problemas imensos que têm. Mas e daí? Só vemos; não podemos fazer nada (...). De que adianta ver o que acontece na comunidade? Não tem como melhorar! Só aconselhar não adianta!".

A fala, enunciada em tom de desabafo, expressa sentimentos de angústia e impotência frente ao contato com a vida social dos usuários. Mas demonstra também a lógica implícita do modelo de organização da atenção básica. A etnografia dos cuidados prestados pelos profissionais de nível superior, particularmente os médicos, mostrou que eles se estruturam em torno da demanda espontânea e dos sintomas atuais, valorizando apenas aqueles passíveis de tratamento medicamentoso. A observação mostrou que, mesmo para as doenças crônicas cujo cuidado pressupõe uma longa convivência entre doente e profissional de saúde, o tipo de atendimento prestado persiste orientado para a queixa atual e não para o seguimento longitudinal do usuário. A lógica do atendimento pontual obscurece a apreensão da temporalidade na produção da doença e dificulta, mesmo no plano biológico, a compreensão das inter-relações dos diversos sistemas corporais progressivamente comprometidos e das mudanças na constelação sintomática à medida que a patologia avança.

É em decorrência dessa perda da totalidade da expressão histórica da doença que um portador de diabetes que se queixa de problemas de visão pode ser rechaçado por vir à unidade no “dia do HIPERDIA". Da mesma forma, não observamos escuta e acolhimento das queixas relativas às crises vitais que acompanham a história pessoal de adoecimento, às limitações físicas e sociais que se instalam e às repercussões da condição de doente crônico na vida cotidiana. Descrença na cura, conflitos familiares e dificuldades financeiras são dimensões do adoecimento que não encontram meios de expressão na consulta. O modo como o cuidado é ofertado elimina a possibilidade de acompanhamento dessas di- 
mensões da vida do doente, comprometendo a integralidade.

A Atenção Básica deve operar como um nível de atenção orientado pelo uso de tecnologias leves 28 , pautadas pela confiança, relacionamento positivo, capacidade de diálogo e de negociação por parte dos profissionais. Porém, o atendimento ambulatorial encontrado nas unidades investigadas tem como parâmetro implícito de ação o modelo emergencial-curativo, mostrando-se incapaz de produzir uma atenção personalizada e de criar vínculo com o paciente. O peso excessivo do componente farmacológico desvaloriza a dimensão relacional do cuidado. Ainda que o medicamento seja peça fundamental no controle da hipertensão e do diabetes, sozinho, ele é insuficiente para garantir uma adesão permanente do doente aos cuidados oferecidos pela unidade de saúde 3 .

Nessas circunstâncias, a autonomia do usuário, o poder de tomar decisões e o direito de aderir ou rechaçar as prescrições são vistos como obstáculos à melhoria da organização dos serviços. Reduzidos à impotência, os profissionais apontam a intensificação do investimento tecnológico como alternativa de aprimoramento da qualidade da atenção e de garantia da adesão dos doentes às suas prescrições. São os meios que buscam para vencer o chamado "problema cultural”, o rótulo sob o qual enquadram os comportamentos recalcitrantes dos usuários.

No âmbito da educação em saúde, destaque-se a limitada habilidade dos profissionais de nível superior, principalmente dos médicos, em orientar a dieta dos pacientes. É uma dificuldade potencializada pelo desconhecimento dos hábitos alimentares da população. A orientação dietética observada limitava-se a listar uma série de alimentos que não poderiam ser consumidos; porém, não ofereciam orientações sobre a dieta adequada. Igualmente, era deficiente a capacidade de escuta dos profissionais em relação às queixas e/ou problemas trazidos pelos pacientes sobre aspectos não biomédicos, mas intervenientes na dieta como, por exemplo, as dificuldades de aquisição dos alimentos mais saudáveis.

Tais achados são compatíveis com um estudo realizado em unidade básica de saúde de Ribeirão Preto que demonstrou que apenas 17,2\% da amostra de pacientes diabéticos receberam orientações adequadas para seu tratamento 29. Um inquérito populacional, realizado no Rio Grande do Sul, mostrou que a adequação das orientações a pacientes hipertensos era diretamente proporcional ao seu lugar no nível mais elevado da escala social 3, ao passo que a educação em saúde, quando realizada no âmbito dos atendimentos individuais, tinha um caráter essencialmente prescritivo 27 , com ênfase na necessidade de uso correto da medicação e no cumprimento da dieta.

A dificuldade de acesso aos níveis de maior complexidade do sistema também compromete a efetividade do cuidado. Ao atender uma idosa com seqüelas de acidente vascular cerebral, o médico da unidade de saúde da família lhe perguntou em que poderia ajudá-la; a senhora respondeu que gostaria de ter um remédio para curar sua perna debilitada. Em retorno, o profissional lhe disse que poderia ofertar apenas um encaminhamento para o especialista; dirigiu-se, em seguida, ao enfermeiro e perguntou: "Como a gente faz para conseguir neurologista?". O enfermeiro respondeu: "Não consegue, manda para o Serviço A ou para o Serviço B. Só quem tem neurologista é a Secretaria Estadual, e eles não aceitam nossos encaminhamentos". O "não conseguir" sintetizava a inexistência de um sistema organizado de referência. O termo "manda" indicava que a paciente seria enviada à procura de atendimento sem qualquer garantia de consegui-lo. Ouvindo esse diálogo, a senhora exclamou indignada: "É por isso que as pessoas não procuram o médico. É muito dificultoso!". O empréstimo da fala dessa usuária para intitular esse artigo se deve à forma sintética como ela expressou as dificuldades enfrentadas pelos pacientes em busca de acesso aos serviços e ao cuidado integral para suas necessidades de saúde.

\section{Conclusões}

A utilização da etnografia nesta pesquisa avaliativa, tendo o HIPERDIA como condição traçadora, mostrou-se uma experiência exitosa. A observação direta e comparativa do cotidiano das unidades de saúde com e sem-PSF trouxe à tona as rotinas desenvolvidas nas mesmas, indo além do discurso dos pacientes e dos profissionais. A eleição do HIPERDIA como condição traçadora possibilitou a análise não só das características desse programa, mas da própria organização da Atenção Básica em Manaus.

A potencialidade do PSF em facilitar o acesso da população aos serviços de saúde é inviabilizada pela baixa cobertura do programa e pela precariedade da infra-estrutura urbana das áreas mais carentes. A inexistência de um efetivo sistema de referência e contra-referência torna-se uma barreira, por vezes intransponível, para o acesso dos usuários a cuidados de maior nível de complexidade. Na unidade básica de saúde, com maior capacidade instalada, mas que não opera em bases territoriais, observa-se uma situação inversa; nela, encontrou-se maior facili- 
dade de acesso a exames e a certos especialistas e uma menor facilidade de entrada no serviço. Pode-se afirmar também que as unidades com e sem-PSF operavam de forma desarticulada, com fragmentação do cuidado e desconhecimento das dimensões sociais do adoecimento. A maior capacidade instalada da unidade básica de saúde não era aproveitada em favor dos usuários das unidades com PSF mesmo quando havia proximidade geográfica.

Na unidade de saúde da família e na unidade básica de saúde, o cuidado com as demandas de saúde estava centrado na doença, pautando-se pelo enfoque individual e medicalizador, com pouca penetração das atividades de educação em saúde no cotidiano das práticas sanitárias. A proximidade da equipe (ou de parte dela, como o programa agentes comunitários de saúde da unidade básica de saúde) com a comunidade permitia que o conhecimento da realidade social fosse acessível a alguns profissionais, sobretudo agentes comunitários de saúde e enfermeiros, ainda que os médicos o desvalorizassem. Tal co-

\section{Resumo}

O texto analisa os resultados de pesquisa avaliativa da Atenção Básica à Saúde do paciente com hipertensão elou diabetes em Manaus, Amazonas, Brasil. A abordagem etnográfica utilizou, como categorias analíticas centrais, o acesso aos serviços e a integralidade do cuidado, comparando-se as práticas sanitárias desenvolvidas em unidade do Programa Saúde da Família (PSF) e em unidade básica de saúde não-PSF. A facilitação do acesso à unidade de saúde da família implantada em comunidade carente é limitada pela precariedade de infra-estrutura urbana do seu entorno. A unidade básica de saúde tem, nas grandes distâncias, a sua principal barreira de acesso. A inexistência de sistema de referência entre os distintos níveis de complexidade compromete o acesso dos pacientes a exames e especialistas. O cuidado oferecido nas duas unidades é restrito às queixas físicas passivveis de abordagem farmacológica, comprometendo a integralidade. Há baixa capacidade de escuta dos profissionais para problemas distintos do foco da ação programática. Destacam-se as potencialidades da utilização da etnografia na pesquisa avaliativa de sistemas e serviços de saúde.

Atenção Primária à Saúde; Antropologia Cultural; Hipertensão; Diabetes Mellitus nhecimento gerava, por vezes, sentimentos de angústia e impotência, mas não se observou a produção de estratégias que propiciassem a incorporação das informações sobre as condições de vida nos processos de trabalho desenvolvidos nas unidades.

As categorias analíticas centrais da pesquisa (acesso e integralidade) mostraram-se ferramentas eficientes na comparação e análise da oferta de cuidados em unidade básica de saúde nãoPSF e em unidade de saúde da família. Os resultados da investigação não mostraram diferença significativa entre elas no que tange à qualidade e integralidade do cuidado ofertado. Ambas partilhavam baixa capacidade de responsabilização, de vínculo, escuta e acolhimento de problemas que fugissem do foco imediato da ação programática e mesmo de ações não-medicamentosas previstas nas rotinas do HIPERDIA. Os resultados da investigação reforçam a idéia de que há um longo caminho a ser percorrido na qualificação da Atenção Básica, em busca de alcance pleno dos princípios que regem o SUS.

\section{Colaboradores}

L. Garnelo e M. L. P. Souza participaram conjuntamente da concepção do artigo, da análise nele contida e da revisão do texto final.

\section{Agradecimentos}

Os autores agradecem ao Dr. Eronildo Felisberto, ao Departamento de Atenção Básica, do Ministério da Saúde e às Dras. Maria Helena Mendonça e Maria do Carmo Leal, da Escola Nacional de Saúde Pública, Fundação Oswaldo Cruz, pelo apoio institucional para realização desta pesquisa. Pesquisa financiada pelo Ministério da Saúde, no âmbito do projeto Monitoramento e Avaliação do Programa de Expansão e Consolidação da Saúde da Família (PROESF), para o Desenvolvimento de Estudos Avaliativos - Linhas de Base - de Municípios. 


\section{Referências}

1. Firmo JOA, Lima-Costa MF, Uchoa E. Projeto Bambuí: maneiras de pensar e de agir de idosos hipertensos. Cad Saúde Pública 2004; 20:1029-40.

2. Paiva DCP, Bersusa AAS, Escuder MML. Avaliação da assistência ao paciente com diabetes e/ou hipertensão pelo Programa Saúde da Família do Município de Francisco Morato, São Paulo, Brasil. Cad Saúde Pública 2006; 22:377-85.

3. Sturmer G, Dias-da-Costa JS, Olinto MTA, Menezes AMB, Gigante DP, Macedo S. O manejo não medicamentoso da hipertensão arterial sistêmica no Sul do Brasil. Cad Saúde Pública 2006; 22:1727-37.

4. Assunção MCF, Santos IS, Costa JSD. Avaliação do processo de atenção médica: adequação do tratamento de pacientes com diabetes mellitus, Pelotas, Rio Grande do Sul, Brasil. Cad Saúde Pública 2002; 18:205-11.

5. Secretaria de Políticas Públicas, Ministério da Saúde. Plano de Reorganização da Atenção à Hipertensão Arterial e ao Diabetes Mellitus. Rev Saúde Pública 2001; 35:585-8.

6. Ayres JRCM. Hermenêutica e humanização das práticas de saúde. Ciênc Saúde Coletiva 2005, 10:549-60.

7. Senna MCM. Eqüidade e política de saúde: algumas reflexões sobre o Programa Saúde da Família. Cad Saúde Pública 2002; 18 Suppl:S203-11.

8. Ribeiro JM, Costa NR, Pinto LFS, Silva PLB. Atenção ao pré-natal na percepção das usuárias do Sistema Único de Saúde: um estudo comparativo. Cad Saúde Pública 2004; 20:534-45.

9. Ministério da Saúde. Saúde da família: uma estratégia para a reorientação do modelo assistencial. Brasília: Ministério da Saúde; 1998.

10. Contandriopoulos AP, Champgne F, Denis JL, Pineault R. A avaliação na área de saúde: conceitos e métodos. In: Hartz ZMA, organizador. Avaliação em saúde: dos modelos conceituais à prática na análise da implantação de programas. Rio de Janeiro: Editora Fiocruz; 2000. p. 29-48.

11. Clifford J. A experiência etnográfica. Antropologia e literatura no século XX. Rio de Janeiro: Editora da UFRJ; 2002.

12. Dezin NK, Lincoln YS. Introduction: the discipline and practice of qualitative research. In: Dezin NK, Lincoln YS, editors. Handbook of qualitative research. Thousand Oaks: Sage Publications; 2000. p. 1-29.

13. Deslandes SF, Gomes R. A pesquisa qualitativa nos serviços de saúde: notas teóricas. In: Bosi MLM Mercado FJ, organizadores. Pesquisa qualitativa de serviços de saúde. Petrópolis: Editora Vozes; 2004. p. 99-120.

14. Bourdieu PA. Economia das trocas lingüísticas. São Paulo: Edusp; 1998.

15. Travassos C, Martins M. Uma revisão sobre os conceitos de acesso e utilização de serviços de saúde. Cad Saúde Pública 2004; 20 Suppl 2:S190-8.
16. Mattos RA. Os sentidos da integralidade: algumas reflexões acerca de valores que merecem ser defendidos. In: Pinheiro R, Mattos RA, organizadores. Os sentidos da integralidade na atenção e no cuidado à saúde. Rio de Janeiro: Instituto de Medicina Social, Universidade do Estado do Rio de Janeiro/ABRASCO; 2001. p. 39-64

17. Merhy EE. Saúde: a cartografia do trabalho vivo. São Paulo: Editora Hucitec; 2005.

18. Geertz C. A interpretação das culturas. Rio de Janeiro: Livros Técnicos e Científicos Editora; 1989.

19. Coelho EB, Moysés Neto M, Palhares R, Cardoso MCM, Geleilete TJM, Nobre F. Relação entre a assiduidade às consultas ambulatoriais e o controle da pressão arterial em pacientes hipertensos. Arq Bras Cardiol 2005; 85:157-61.

20. Araújo RB, Santos I, Cavaleti MA, Costa JSD, Béria JU. Avaliação do cuidado prestado a pacientes diabéticos em nível primário. Rev Saúde Pública 1999; 33:24-32.

21. Monteiro MF, Sobral-Filho DC. Exercício físico e o controle da pressão arterial. Rev Bras Med Esporte 2004; 10:513-6.

22. Maciel ICF, Araújo TL. Consulta de enfermagem: análise das ações junto a programas de hipertensão arterial, em Fortaleza. Rev Latinoam Enferm 2003; 11:207-14.

23. Raskin DF, Pinto-Neto AM, Paiva LHSC, Raskin A, Martinez EZ. Fatores associados à obesidade e ao padrão andróide de distribuição da gordura corporal em mulheres climatéricas. Rev Bras Ginecol Obstet 2000; 22:435-41.

24. Mac Fadden MAJ, Ribeiro AV. Aspectos psicológicos e hipertensão essencial. Rev Assoc Med Bras 1998; 44: 4-10.

25. Andreatini R. Uso de fitoterápicos em psiquiatria. Rev Bras Psiquiatr 2000; 22:104-5.

26. Sakata K, Bigolin S, Bryk Junior A, Komatsu MCG, Sakata L, Vanzo LRC, et al. Estudo dos conhecimentos de pacientes com hipertensão, diabetes ou glaucoma sobre suas doenças. Arq Bras Oftalmol 2002; 65:467-9.

27. Alves VS, Nunes MO. Educação em saúde ao paciente com hipertensão arterial no Programa Saúde da Família. Comun Saúde Educ 2006; 9:131-47.

28. Merhy EE. Em busca do tempo perdido: a micropolítica do trabalho vivo em saúde. In: Merhy EE, Onocko R, organizadores. Agir em Saúde. Um desafio para o público. São Paulo: Editora Hucitec; 1997. p. 71-112.

29. Guimarães FPM, Takayanagui AMM. Orientações recebidas do serviço de saúde por pacientes para o tratamento do portador de diabetes mellitus tipo 2. Rev Nutr 2002; 15:37-44.

Recebido em 22/Fev/2007

Versão final reapresentada em 05/Jul/2007

Aprovado em 13/Jul/2007 